\title{
The Uriscreen test was not better than standard urinalysis and dipstick tests for detecting urinary tract infection in children
}

\author{
Waisman Y,Zerem E, Amir L, et al. The validity of the Uriscreen test for early detection of urinary tract infection in \\ children. Pediatrics 1999 Oct;104:e41.
}

QUESTION: Is the Uriscreen test better than standard urinalysis and dipstick tests for early detection of urinary tract infection (UTI) in children?

Design

Independent comparisons of Uriscreen, urinalysis, and dipstick tests with quantitative urine culture.

\section{Setting}

Emergency department of a children's hospital in Israel.

\section{Participants}

A random sample of 121 children (68\% girls) who were 1 month to 17 years of age and who presented to the emergency department with symptoms of UTI. Symptoms for infants were fever with no apparent source, vomiting, decreased appetite, and irritability; for toddlers, abdominal pain and frequent voiding with or without fever; and for older children, dysuria, frequency, urgency, and abdominal or flank pain with or without fever. The exclusion criterion was use of antibiotics.

\section{Description of tests and diagnostic standard}

A urine sample was obtained from each child by using age appropriate methods. Within 15 minutes of collection, urine samples were tested in 4 ways. The Uriscreen test was a commercial kit (Diathec Diagnostica, Rehovot, Israel; cost US $\$ 0.71$ per test); the dipstick test was the Multistix 10 SG strip (Bayer, Elkhart, IN, USA; cost US $\$ 0.27$ per test); and urinalysis was done in the hospital laboratories with an automated urine analyser (Urotron RL9, Boehringer Mannheim; cost US $\$ 13$ per test). The diagnostic standard was quantitative urine culture, which was done by using the commercial Diaslide method (Diathec Diagnostica, Rehovot, Israel).

Main outcome measures

Sensitivity and specificity for detecting UTI.

\section{Main results}

5 of 39 boys (13\%) and 30 of 82 girls (37\%) had UTIs.

Source of funding: no external funding.

For correspondence: Dr Y Waisman, Unit of Emergency Medicine, Schneider Children's Medical Center of Israel, 14 Kaplan Street, Petah Tiqua, Israel 49202. Fax + 97239223011.
The table shows sensitivities, specificities, and likelihood

Test characteristics for early detection of urinary tract infection in children*

\begin{tabular}{lcccl} 
Tests & Sensitivity $(95 \%$ Cl) & Specificity (CI) & +LR & -LR \\
Uriscreen & $100 \%(90$ to 100$)$ & $69 \%(58$ to 78$)$ & 3.2 & $\infty$ \\
\hline Dipstick & $97 \%(85$ to 100$)$ & $83 \%(73$ to 90$)$ & 5.6 & 0.03 \\
\hline Urinalysis & $89 \%(73$ to 97$)$ & $88 \%(80$ to 94$)$ & 7.6 & 0.13 \\
\hline
\end{tabular}

* LRs and $\mathrm{Cl}$ defined in glossary and calculated from data in article. ratios. The Uriscreen test had perfect sensitivity but low specificity and was not better than the dipstick or urinalysis tests for detecting UTI early.

\section{Conclusion}

The Uriscreen test was not better than dipstick or urinalysis tests for the early detection of urinary tract infection in children.

\section{COMMENTARY}

The Uriscreen test is a new method for rapid diagnosis of UTI that tests for catalase. Catalase is produced by bacteria and cells and is generally absent if there are no leukocytes, erythrocytes, or bacteria in the urine. Studies of Uriscreen in adults have shown mixed results. A previous study in children found the test's sensitivity too low to be useful for screening, ${ }^{1}$ whereas the study by Waisman et al found that the test had a high sensitivity but low specificity in symptomatic children.

Why have these and other studies differed regarding the accuracy of Uriscreen? One possibility is spectrum bias (ie, differences in sensitivity and specificity of a test that result from differences in how the disease is manifested in a population). ${ }^{2}$ Differences in disease features between symptomatic and asymptomatic patients could be expected, and differences in how the cultures are interpreted can explain some differences in test performance. Culture results are affected by the source of the urine, the number of colony forming units that are considered evidence of infection, and the interpretation of cultures that show multiple organisms.

What should the practitioner do? Test errors in UTI are asymmetrical: a false negative result that leads to an infected patient not receiving treatment can be more important than a false positive result. Thus, the high sensitivity found in this study suggests it is useful. We need more definitive knowledge of Uriscreen's performance under different conditions and with different populations, however, before physicians can use it with confidence for deciding about treatment.

Robert S Wigton, MD, MS University of Nebraska Medical Center Omaha, Nebraska, USA

1 Palmer LS, Richards I, Kaplan WE. Clinical evaluation of a rapid diagnostic screen (URISCREEN) for bacteriuria in children.J Urol 1997;157:654-7.

2 Lachs MS, Nachamkin I, Edelstein PH, et al. Spectrum bias in the evaluation of diagnostic tests: lessons from the rapid dipstick test for urinary tract infection. Ann Intern Med 1992;117:135-40. 